\title{
Mesoscopic lattice modeling of electrokinetic phenomena
}

\author{
Ignacio Pagonabarraga ${ }^{\mathrm{a}, *}$, Fabrizio Capuani $^{\mathrm{b}}$, Daan Frenkel ${ }^{\mathrm{b}}$ \\ a Departament de Física Fonamental, Universitat de Barcelona, Carrer Martí i Franqués 1, 08028-Barcelona, Spain \\ ${ }^{\mathrm{b}}$ Instituut Amolf, Kruislaan 407, 1098SJ Amsterdam, The Netherlands
}

Available online 12 April 2005

\begin{abstract}
The development of models for electrolytes is challenging due to the long-range nature of electric interactions. We propose a novel implementation of a lattice Boltzmann model that solves a number of limitations and inconsistencies in previously proposed variants and discuss the range of parameters the model can cover. We show how the relevant electrohydrodynamic couplings are recovered analyzing a particular electrokinetic phenomena.

(c) 2005 Elsevier B.V. All rights reserved.
\end{abstract}

PACS: 47.65.+a; 47.11.+j; 05.20.Jj; 82.45.Gj

Keywords: Electrohydrodynamics; Kinetic models; Electroosmosis

\section{Introduction}

Complex fluids are characterized by the coexistence of a variety of length scales and their corresponding time scales [1]. For example, in a colloidal suspension, solvent molecules (of size up to a few angstroms), coexist with suspended solid particles of sizes between tens and hundreds of nanometers. The need to cover the relevant colloidal time scales requires coarse-graining. The use of kinetic models (e.g., lattice Boltzmann (LB) [2]), direct simulation Monte Carlo [3] or stochastic rotation dynamics [4], among others keeps the relevant collective modes of the solvent, and their coupling to the dynamics of

\footnotetext{
* Corresponding author.

E-mail address: ipagonabarraga@ub.edu (I. Pagonabarraga).
}

the mesoscopic objects which are suspended in equilibrium. They offer an alternative approach to other mesoscopic techniques, which disregard the proper dynamics of the solvent, as in Brownian Dynamics [5], or which recover them only on specific limits (e.g., Stokesian Dynamics [6]).

It has been recognized that LB captures the hydrodynamics of colloidal suspensions efficiently and it has been used to study the short and long time diffusion of suspensions [7], or to understand quantitatively confinement effects on its collective behavior [8]. Although most of the numerical studies have been performed on hard-core colloids, recently there has been interest in considering the effect of electrolyte and charge in colloid hydrodynamics within LB. Modeling electrolytes poses additional challenges, because it is necessary to ensure strict local charge density conser- 
vation; otherwise the long-range nature of electrostatic interactions may give rise to uncontrolled errors [9]. In Section 2 we describe the peculiarities of the LB model we propose to solve previously found limitations and we discuss some of its technical peculiarities and scope in Section 3. We also analyze a particular example where electrohydrodynamic couplings are present, and conclude summarizing the main results of the paper.

\section{Numerical model}

LB is based on the particle density moving in direction $\mathbf{c}_{i}$ at time $t$ from node $\mathbf{r}, f\left(\mathbf{r}, \mathbf{c}_{i}, t\right)$. The lattice on which $f\left(\mathbf{r}, \mathbf{c}_{i}, t\right)$ evolve is defined by its dimensionality and connectivity, the latter prescribed by the subset of allowed velocities, $\left\{\mathbf{c}_{i}\right\}$. The dynamics is defined by a relaxation step, in which $f\left(\mathbf{r}, \mathbf{c}_{i}, t\right)$ relax towards a prescribed equilibrium distribution (which corresponds to a discretized version of the linearized collision operator of the Boltzmann equation) and an advection step where they move from node $\mathbf{r}$ to node $\mathbf{r}+\mathbf{c}_{i}$; hence the dynamics is local and discrete both in space and time, one of the major computational advantages of LB. Velocity moments of $f\left(\mathbf{r}, \mathbf{c}_{i}, t\right)$ are related to hydrodynamic variables and Navier-Stokes equation is recovered on appropriate length and time scales [2]. Non-ideality is incorporated either by modifying the collision rule $[10,11]$ or by relating the equilibrium distribution and pressure tensor to a freeenergy model [12].

Instead of describing the molecular nature of the electrolyte, or deriving its dynamics from a kinetic equation, we describe the electrolyte by local densities, $\rho_{+}(\mathbf{r}, t)$ and $\rho_{-}(\mathbf{r}, t)$ on each lattice node. Consistent with this approach, we assume that its dynamics is diffusive (since inertia is negligible). To account for the diffusive relaxation of the local electrolyte densities, we introduce as the basic quantity the diffusive flux of species along each allowed link, $j_{ \pm i}$, which can be expressed as

$$
\begin{aligned}
j_{ \pm i}(\mathbf{r})= & -d_{ \pm} \frac{\mathrm{e}^{\mp z_{ \pm} \Delta \Phi(\mathbf{r})}+1}{2} \\
& \times\left[\frac{\rho_{ \pm}\left(\mathbf{r}+\mathbf{c}_{i}\right)-\rho_{ \pm}(\mathbf{r}) \mathrm{e}^{ \pm z_{ \pm} \Delta \Phi(\mathbf{r})}}{\Delta_{i}}\right],
\end{aligned}
$$

where $\Delta \Phi(\mathbf{r}) \equiv \Phi(\mathbf{r})-\Phi\left(\mathbf{r}+\mathbf{c}_{i}\right), d_{ \pm}$is related to the diffusivity of the ions, $\Delta_{i}=\left|\mathbf{c}_{i}\right|$ is the distance between the two neighboring nodes, $z_{ \pm}$is the valency of the corresponding species and $\Phi(\mathbf{r})$ a dimensionless electrostatic potential which satisfies the Poisson equation,

$\nabla^{2} \Phi=-4 \pi l_{B} \sum_{k= \pm} z_{k} \rho_{k}$

with $l_{B}=\beta \mathrm{e}^{2} /(4 \pi \epsilon)$ the Bjerrum length (the distance at which electrostatic and thermal energy are equal). In the presence of charged solids, the corresponding charge density should be added to the r.h.s. of Eq. (2). The local density change due to diffusion can then be expressed as

$\rho_{ \pm}(\mathbf{r}, t+1)=\rho_{ \pm}(\mathbf{r}, t)+A_{0} \sum_{i} j_{ \pm i}(\mathbf{r}, t)$,

where $A_{0}$ is an appropriate geometric factor related to the underlying lattice. Due to the symmetric definition of the link fluxes (which ensures that $j_{ \pm i}(\mathbf{r}, t)=$ $-j_{ \pm i^{\prime}}\left(\mathbf{r}+\mathbf{c}_{i^{\prime}}, t\right)$ if $\left.\mathbf{c}_{i}=-\mathbf{c}_{i^{\prime}}\right)$, the rule (3) does ensure that charge is strictly conserved locally. Since $f\left(\mathbf{r}, \mathbf{c}_{i}, t\right)$ defines a local velocity, $\mathbf{v}(\mathbf{r})$, at each time step one needs to advect the charge densities with that velocity. This is achieved by virtually displacing node $\mathbf{r}$ a quantity $\mathbf{v}(\mathbf{r})$, and distributing the charges to the neighboring nodes proportionally to the overlap between the virtually displaced node and the neighboring nodes. It has been shown that this method recovers the desired convection diffusion equation for the charged species on appropriate length and time scales [13],

$\frac{\partial \rho_{ \pm}}{\partial t}+\mathbf{v} \cdot \nabla \rho_{ \pm}=D_{ \pm} \nabla \cdot\left[\nabla \rho_{ \pm} \pm z_{ \pm} \rho_{ \pm} \nabla \Phi\right]$,

where the diffusion coefficient satisfies, $D_{ \pm}=A_{0} d_{k}$ ( $A_{0}$ will depend on the lattice on which LB is defined; e.g., for a cubic lattice with eighteen velocities, $A_{0}=1+2 \sqrt{2}$ ). Advection induces an additional spurious diffusivity, which leads to an effective diffusion coefficient,

$$
\begin{aligned}
D_{ \pm}^{\mathrm{eff}}= & D_{ \pm}+\frac{1}{2}\left[v_{x}\left(1-v_{x}\right)+v_{y}\left(1-v_{y}\right)\right. \\
& \left.+v_{z}\left(1-v_{z}\right)\right] .
\end{aligned}
$$

Since the velocity is necessarily smaller than 1 in lattice units [14] the magnitude of the relative error in the effective diffusion is kept below $5 \%$, as illustrated 


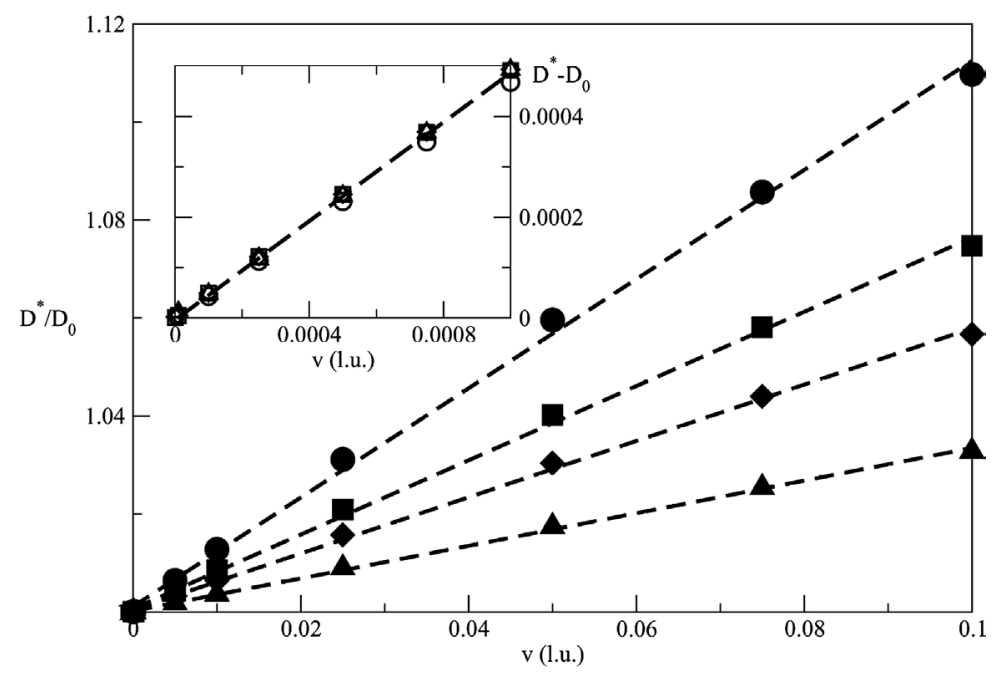

Fig. 1. Effective diffusion coefficient, $D^{*}$ (measured in units of the reference diffusion $D_{0}=D_{+}=D_{-}$for a symmetric electrolyte) as a function of the velocity. In the simulations a uniform velocity is imposed, and the steady charge profile that develops due to a semipermeable membrane that is impermeable to the electrolyte is measured. From the profile the effective diffusion coefficient is extracted.

in Fig. 1. The inset shows that the induced-flow diffusion does not depend on its equilibrium value, $D_{0}$, and has a linear dependence in the flow velocity for small velocities.

Advancing together Eqs. (3) and (2) [15], provides a way to simulate the convection-diffusion equation that characterizes the collective behavior of charge densities when molecular details and inertia are negligible.

Global charge electroneutrality allows for a local buildup of charge; in those cases, a force will act on the solvent. Such a force is incorporated modifying the lattice-Boltzmann equation accordingly [7]. The electric force can be composed out of the link fluxes [13], which ensures that the force and diffusive fluxes are numerically consistent with each other. It has been shown that such an implementation eliminates spurious fluxes that do feature in other non-ideal LB models.

In the presence of solid interfaces which appear when dealing, e.g., with fluids in the presence of solid walls, or when modeling colloidal suspensions (colloids are usually treated as solid surfaces where bounce-back is imposed on the densities $f\left(\mathbf{r}, \mathbf{c}_{i}, t\right)$ [7]) it is straightforward to implement the no flux boundary condition for the charge densities: it is enough to state that the corresponding link flux, $j_{ \pm i}$, is zero, and during the advection step to prevent moving charges into solid nodes. One can hence bypass some of the limitations in previous LB implementations.

\section{Diffusivities and hydrodynamic coupling}

The proposed LB can span a wide range of $D_{ \pm}$. We have checked that values between $10^{-3}$ and 1 (in lattice units) are feasible and provide accurate results. From a numerical point of view, accessing large values of the diffusion should be done with care because a too large diffusive link flux may produce locally oscillating densities. To avoid that, we have decomposed the diffusive step into $N_{\text {it }}$ substeps, and in each substep a fraction $1 / N_{\text {it }}$ of the link fluxes is transported. Effectively we have a diffusion $d_{ \pm} / N_{\text {it }}$ which allows for a gradual rearrangement of matter so that after the $N_{\text {it }}$ iterations the overall proper amount of electrolyte has been diffused avoiding any numerical instability. By considering a localized density variation, one can ensure absolute stability for $d_{\mathrm{thr}} \leqslant 1 /(6(1+2 \sqrt{2}))$. Accordingly, $d_{ \pm} / d_{\text {thr }}$ provides a good estimate for $N_{\mathrm{it}}$.

In LB the kinematic viscosity, $v$, can span a wide range of values (viscosities of order 1/2 (lattice units) are regarded as corresponding to a very high viscosity fluid, and $v$ several orders of magnitude smaller are accessible). As a result, a wide variety of Schmidt numbers, $S c=v / D_{ \pm}$, can be achieved. For fluids one 

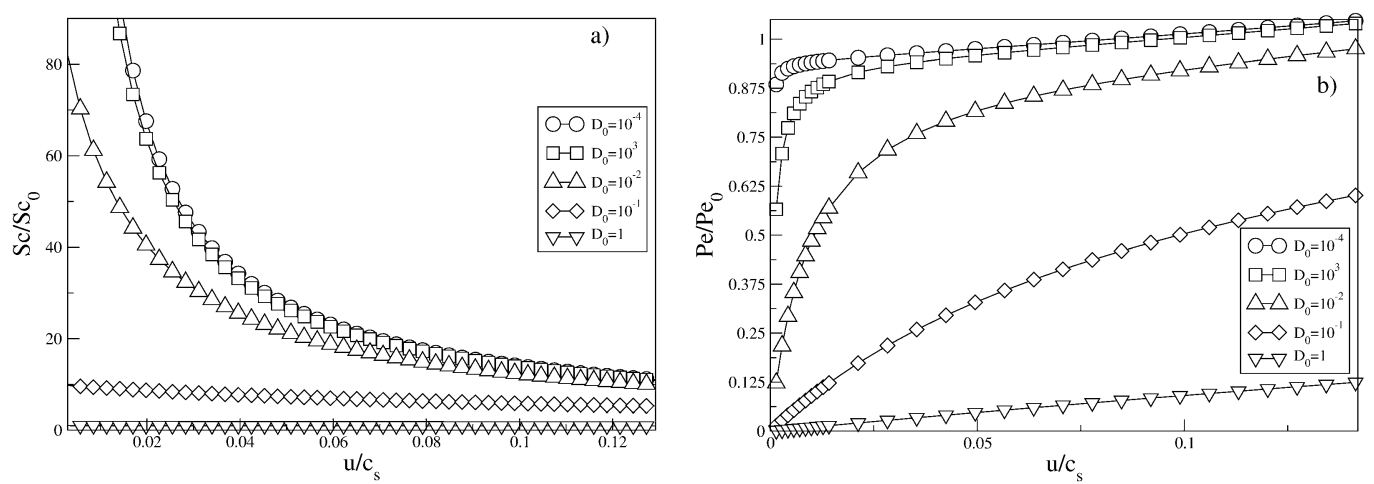

Fig. 2. Schmidt and Péclet number for a symmetric electrolyte ( $D_{0}=D_{+}=D_{-}$). The effective diffusivity is estimated assuming the three components of the velocity are equal. (a) Schmidt number normalized by $S c_{0}=1$, as a function of the local velocity for different reference diffusivities. (b) Péclet number normalized by the Péclet which corresponds to a particle of radius 1 , moving at $c_{S}$, as a function of the local velocity for different reference diffusivities.

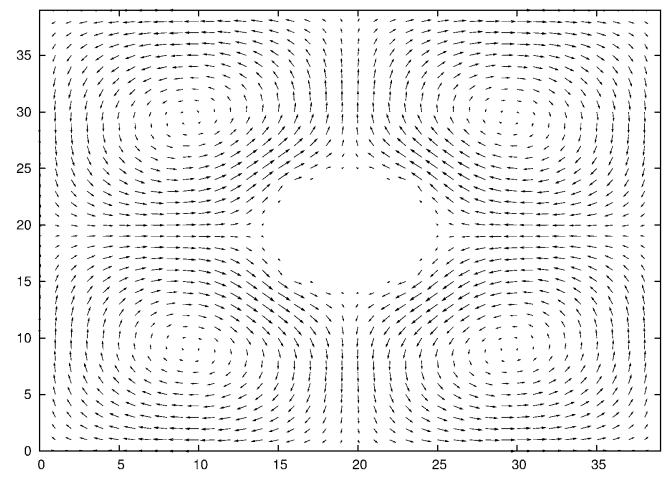

(a)

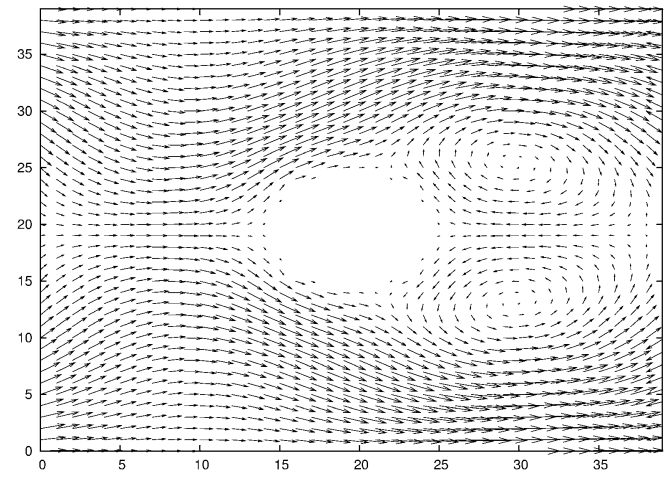

(b)

Fig. 3. Velocity field around a sphere of radius 4.5 (white circle) under an applied field which corresponds to an applied voltage of 0.1 along the $x$-axis. (a) Neutral sphere with an electric dipole parallel to the applied field; (b) a negative charge is added to the previous sphere to break the symmetry of the flow field (see Fig. 1 in Ref. [17] to compare the streamlines).

is interested in $S c>1$, which can be achieved easily by combining different values for $v$ and $D_{ \pm}$; the regime of very high $S c$ is more difficult to reach in the presence of an externally imposed flow (when the effective diffusion increases). In Fig. 2(a) we show that, except for large $D_{ \pm}$, large values for $S c$ are achieved, despite its sensitivity to local flows due to spurious diffusion.

If one is interested in the dynamics of colloidal suspensions (or other mesoscopic particles) it is important to be able to control the Péclet number, $P e=u R / D_{ \pm}$, which compares the relevance of convective motion on scales of the colloidal particle (characterized by its radius $R$ ) moving at a characteristic velocity $u$ to electrolyte diffusion. In Fig. 2(b) we display the Péclet numbers as a function of the velocity, for various
$D_{ \pm}$. Pe larger than unity are achievable mostly by increasing the particle size. However, since system sizes are limited below $500^{3}$ lattice spacings [16], particle radius above a few tens become unfeasible; as a result, it becomes difficult to get $P e$ above a few tens. Very high $P e$ (the regime where electrolyte diffusion is negligible, relevant when high external fields are applied) are out of reach on the current implementation, mostly because of the spurious diffusivity that rises in the advection step.

In electrokinetic phenomena it is crucial to capture all the relevant couplings between hydrodynamic and electric forces to recover the correct kinetics of suspended particles. For example, it has been recently pointed out that the interaction of an electric imposed 
field with the double layer it creates gives rise to nonlinear electroosmotic flows (induced charged electroosmosis (ICEO) [17]). A simple example of such a phenomena takes place when a neutral sphere with a dipolar charge distribution is placed in a fluid under an applied field. The field induces a symmetric flow field, as shown in Fig. 3(a); symmetry broken if the particle has a net charge, Fig. 3(b) (see Ref. [17], Fig. 1(c) and 1(d) to compare streamlines). Also for sedimentation it has been shown that the proposed model is able to recover all the physically relevant couplings [13] for colloids of various shapes [18].

\section{Conclusions}

We have described the new features of a recently proposed LB for electrolytes. By an appropriate introduction of link fluxes we avoid the persistence of spurious fluxes in equilibrium, as well as at fluid/solid boundaries. An analysis of the range of parameters that can be reached shows that the method is flexible and powerful, as we have illustrated by indicating how all relevant electrohydrodynamic couplings are properly accounted for.

\section{References}

[1] M.E. Cates, M.R. Evans (Eds.), Soft and Fragile Matter, Institute of Physics Pub., Bristol, 2000.
[2] S. Succi, The Lattice Boltzmann Equation for Fluid Dynamics and Beyond, Clarendon Press, Oxford, 2001.

[3] G.A. Bird, Molecular Gas Dynamics, Oxford University Press, Oxford, 1976.

[4] A. Malevanets, R. Krapal, Europhys. Lett. 44 (1998) 552.

[5] M. Allen, D. Tildesley, Computer Simulations of Liquids, Oxford Univ. Press, Oxford, 2000.

[6] G. Bossis, J.F. Brady, Ann. Rev. Fluid Mech. 20 (1988) 111.

[7] A.J.C. Ladd, R. Verberg, J. Stat. Phys. 104 (2001) 1191.

[8] I. Pagonabarraga, Novel Methods in Soft Matter Simulations, in: M. Kartunnen, I. Vattulainen, A. Lukkarinen (Eds.), Lecture Notes in Physics, vol. 640, Springer-Verlag, Berlin, 2004.

[9] J. Horbach, D. Frenkel, Phys. Rev. E 64 (2001) 061507.

[10] X. Shan, H. Chen, Phys. Rev. E 47 (1993) 1815.

[11] L.-S. Luo, Phys. Rev. Lett. 81 (1998) 1618.

[12] M.R. Swift, W.R. Osborn, J.M. Yeomans, Phys. Rev. Lett. 75 (1995) 830.

[13] F. Capuani, I. Pagonabarraga, D. Frenkel, J. Chem. Phys. 121 (2004) 973.

[14] To avoid instabilities and numerical artifacts the velocity should be smaller than 0.1. LB is reliable for subsonic flows, and the speed of sound, e.g. in D3Q18 is $c_{s}=1 / \sqrt{2}$.

[15] We have implemented a standard version of the successive overrelaxation technique (SOR) to solve the Poisson equation, see, e.g., W.H. Press, B.P. Flannery, S.A. Teukolsky, W.T. Vetterling, Numerical Recipes, Cambridge Univ. Press, Cambridge, 1989, because it does not presume periodic boundary conditions, and hence provides a flexible tool for generic geometries. Depending on the situation of interest, alternative strategies may be needed.

[16] J.C. Desplat, I. Pagonabarraga, P. Bladon, Comput. Phys. Comm. 134 (2001) 273.

[17] M.Z. Bazant, T.M. Squires, Phys. Rev. Lett. 92 (2004) 066101.

[18] F. Capuani, I. Pagonabarraga, D. Frenkel, Preprint. 